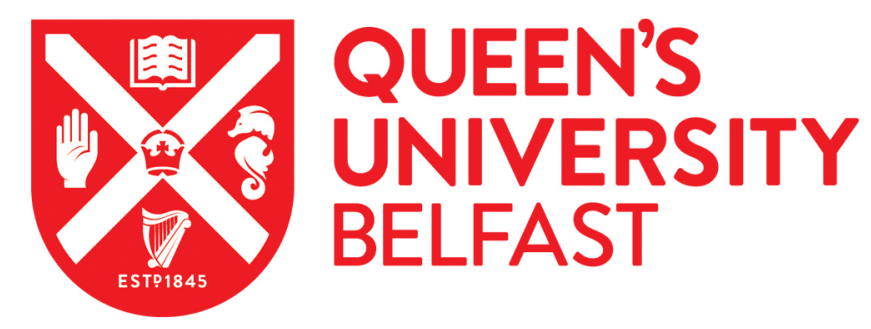

\title{
Scaling laws for laser-driven ion acceleration from nanometer-scale ultrathin foils
}

Shen, X. F., Qiao, B., Pukhov, A., Kar, S., Zhu, S. P., Borghesi, M., \& He, X. T. (2021). Scaling laws for laserdriven ion acceleration from nanometer-scale ultrathin foils. Physical review. E, 104, [025210]. https://doi.org/10.1103/PhysRevE.104.025210

Published in:

Physical review. E

Document Version:

Publisher's PDF, also known as Version of record

Queen's University Belfast - Research Portal:

Link to publication record in Queen's University Belfast Research Portal

Publisher rights

Copyright, 2021 American Physical Society.

This work is made available online in accordance with the publisher's policies. Please refer to any applicable terms of use of the publisher.

\section{General rights}

Copyright for the publications made accessible via the Queen's University Belfast Research Portal is retained by the author(s) and / or other copyright owners and it is a condition of accessing these publications that users recognise and abide by the legal requirements associated with these rights.

Take down policy

The Research Portal is Queen's institutional repository that provides access to Queen's research output. Every effort has been made to ensure that content in the Research Portal does not infringe any person's rights, or applicable UK laws. If you discover content in the Research Portal that you believe breaches copyright or violates any law, please contact openaccess@qub.ac.uk. 


\title{
Scaling laws for laser-driven ion acceleration from nanometer-scale ultrathin foils
}

\author{
X. F. Shen $\odot,{ }^{1,2}$ B. Qiao $\odot,{ }^{1,3,}{ }^{*}$ A. Pukhov,${ }^{2}$ S. Kar $\odot,{ }^{4}$ S. P. Zhu,${ }^{5}$ M. Borghesi,${ }^{4}$ and X. T. $\mathrm{He}^{1,3,5}$ \\ ${ }^{1}$ Center for Applied Physics and Technology, HEDPS, State Key Laboratory of Nuclear Physics and Technology, \\ and School of Physics, Peking University, Beijing 100871, China \\ ${ }^{2}$ Institut für Theoretische Physik I, Heinrich-Heine-Universität Düsseldorf, 40225 Düsseldorf, Germany \\ ${ }^{3}$ Collaborative Innovation Center of IFSA (CICIFSA), Shanghai Jiao Tong University, Shanghai 200240, China \\ ${ }^{4}$ Center for Plasma Physics, School of Mathematics and Physics, Queen's University Belfast, Belfast BT7 1NN, United Kingdom \\ ${ }^{5}$ Institute of Applied Physics and Computational Mathematics, Beijing 100094, China
}

(Received 24 September 2020; revised 20 February 2021; accepted 12 August 2021; published 30 August 2021)

\begin{abstract}
Laser-driven ion acceleration has attracted global interest for its potential towards the development of a new generation of compact, low-cost accelerators. Remarkable advances have been seen in recent years with a substantial proton energy increase in experiments, when nanometer-scale ultrathin foil targets and high-contrast intense lasers are applied. However, the exact acceleration dynamics and particularly the ion energy scaling laws in this novel regime are complex and still unclear. Here, we derive a scaling law for the attainable maximum ion energy from such laser-irradiated nanometer-scale foils based on analytical theory and multidimensional particle-in-cell simulations, and further show that this scaling law can be used to accurately describe experimental data over a large range of laser and target parameters on different facilities. This provides crucial references for parameter design and experimentation of the future laser devices towards various potential applications.
\end{abstract}

DOI: 10.1103/PhysRevE.104.025210

\section{INTRODUCTION}

The study of particle energy scaling law is always a matter of great concern in accelerator physics, especially in laserbased ion accelerators [1-17]. The determination of scaling law is the fundamental basis for the evaluation and optimization of the laser-based acceleration method, in the perspective of developing a competitive alternative to conventional radiofrequency accelerators for applications including medical therapy [18], radiography [19], and fusion energy [20]. Energy scaling laws have been widely investigated and validated for ion acceleration from micron-thick targets [4-11], where target normal sheath acceleration (TNSA) [21] is believed to dominate. In accordance with these scaling laws, the maximum proton energy has increased from $58 \mathrm{MeV}$ [3] to $85 \mathrm{MeV}$ [22] at almost the same laser intensity by decreasing the foil thickness from 70 to $0.9 \mu \mathrm{m}$, with the enhancement attributed to refluxing [23] and concentration [24] of hot electrons for thinner targets. Recent years, owing to progress in target fabrication and laser technologies, nanometer-scale targets with thicknesses from several to tens of nanometers have also been applied in experiments $[13,17,25-30]$, where, besides TNSA, the onset of a theoretically superior acceleration mechanism, namely radiation pressure acceleration (RPA) [31-37], has been observed. Recently, record proton energies of about $94 \mathrm{MeV}$ have been achieved via a hybrid scheme of RPATNSA with the foil thickness reduced to $90 \mathrm{~nm}$ [30]. However, the currently observed maximum energy and energy spread of the ion beams are still far from theoretical predictions $[30,38]$,

\footnotetext{
*bqiao@pku.edu.cn
}

limiting the range of possible applications. In order to guide further performance improvement and optimization of laserion accelerators, the development of accurate and validated scaling laws is an essential step. Yet, a scaling law suitable to describe the maximum ion energy attainable from laserirradiated nanometer-scale foils is still needed, especially in comparison with current experimental results.

In ideal RPA, the scaling law can be described by the "light-sail" (LS) model [31,33], where a nanometer-scale foil is irradiated by a circularly polarized (CP) laser pulse to suppress the $\boldsymbol{j} \times \boldsymbol{B}$ heating. However, in realistic situations, due to multidimensional effects, detrimental transverse instabilities inevitably develop [39-43], and the accelerating foil becomes severely deformed. A significant number of hot electrons are produced and ejected during the interaction, leading to a moving hot electron cloud, and therefore a strong moving electrostatic field is established around the accelerating foil [26]. Because of this, an additional acceleration of ions by this moving sheath field, which we will hereafter refer to as "moving sheath acceleration" (MSA), needs to be taken into account (even for femtosecond laser pulses), as shown in Fig. 1.

In this paper, we theoretically derive the scaling law for the attainable ion-beam maximum energy from laser-irradiated nanometer-scale ultrathin foil targets by considering both above contributions, where the bulk RPA is described by the LS model [33], and the MSA is estimated from the two-phase TNSA model $[5,8]$ in the accelerating foil moving frame and then transferred back to the laboratory frame. The scaling law is verified by our two-dimensional (2D) and three-dimensional (3D) particle-in-cell (PIC) simulations for varying laser and plasma parameters. More importantly, the 


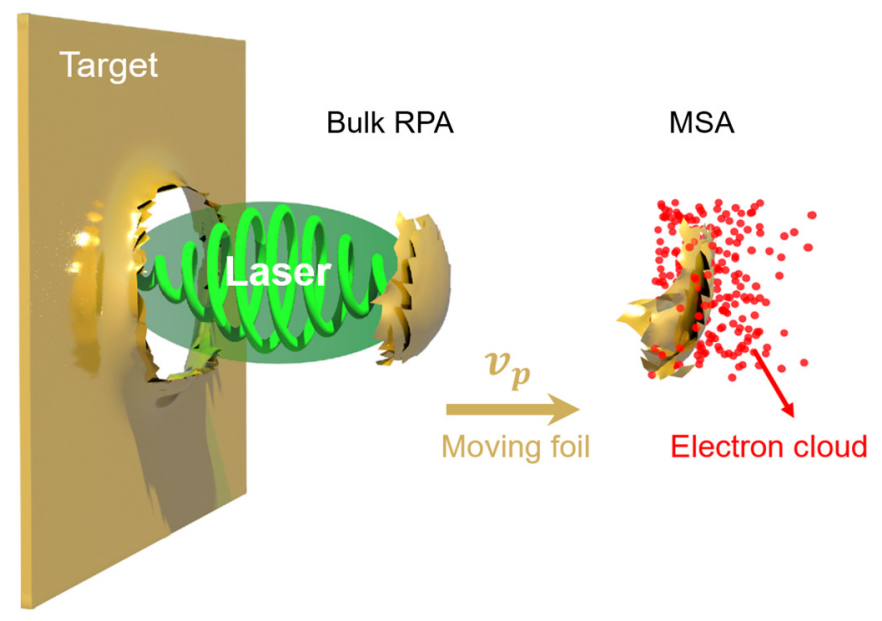

FIG. 1. Schematic of the key acceleration processes from laserirradiated nanofoils: a bulk RPA stage, where a bulk nanofoil (yellow region) is pushed forward by the laser (green), and MSA stage, where high-energy ions are further accelerated by the sheath field in the moving frame, built by hot electrons (red dots).

obtained scaling law is consistent with relevant experimental data from different facilities for a large range of laser and target parameters.

\section{THEORETICAL MODEL}

According to the LS model, ion energy per nucleon $\epsilon_{p}$ gained in an ideal RPA process is given as $\epsilon_{p}=\left[\xi^{2} / 2(1+\right.$ $\xi)] m_{p} c^{2}$, where $\xi=2 \pi(Z / A)\left(m_{e} / m_{p}\right)\left(a_{0}^{2} \tau / \zeta\right), Z / A$ is the charge to mass ratio, $a_{0}$ is the normalized laser intensity, $\tau$ is the normalized pulse duration, and $\zeta=\pi\left(n_{e} / n_{c}\right)(l / \lambda)$. Here, $n_{e}, n_{c}, l$, and $\lambda$ correspond to the initial electron density, critical density, initial target thickness, and laser wavelength, respectively. However, for present tightly focused laser pulses, abundant hot electrons are generated due to transverse instabilities $[39,44]$ and finite spot size effects [26]. On the one hand, this may induce relativistic transparency and terminate the bulk acceleration prematurely; on the other hand, these hot electrons build a strong sheath field around the moving foil, which can further accelerate ions inside the field. In the moving foil frame, this stage of ion acceleration (i.e., MSA) can be described by the adiabatic TNSA model $[5,21]$ as $\epsilon_{s}=(Z / A) \alpha T_{e}$, where $T_{e}$ represents electron temperature and $\alpha$ is a coefficient to be determined according to the specific laser and plasma parameters $[8,21]$. Though MSA starts to function once the sheath field is built, at the beginning, its contribution is always very small compared to that of RPA due to low electron temperature and density. Only near the end of the laser pulse or after transparency, the temperature and density of hot electrons arrive at their maxima, and then MSA dominates the subsequent acceleration. Here, we mainly focused on the case where the bulk acceleration survives throughout the main laser-plasma interaction.

To theoretically give the maximum ion energy obtained from laser-irradiated nm-scale foils, the acceleration process is divided into two stages for simplicity: The first stage is bulk RPA, dominating during the pulse on; the second is MSA, dominating after the pulse over. By a Lorentz transformation, we get

$$
\epsilon_{\max }=\epsilon_{p}+\epsilon_{s}+\frac{\epsilon_{p} \epsilon_{s}}{m_{p} c^{2}}\left(1+\sqrt{1+\frac{2 m_{p} c^{2}}{\epsilon_{p}}} \sqrt{1+\frac{2 m_{p} c^{2}}{\epsilon_{s}}}\right) .
$$

It is obvious that $\epsilon_{\max } \gg \epsilon_{p}$ due to the additional contribution from the MSA process, especially the nonlinear term (the third term) induced by the high speed forward motion of the foil. Therefore, to achieve higher ion energy, a sufficient RPA over the whole laser pulse duration is required, in particular, for a Gaussian laser pulse.

In the foil's reference frame, the sheath field induced by the hot electrons exists at both the front and rear surfaces of the foil $[15,23,45,46]$. The sheath field at the foil rear accelerates ions forward, while that at the front accelerates ions backward, which results in a deceleration of ions when transformed back to the laboratory frame, thus determining the ion minimum energy $\epsilon_{\min }$. The energy spread can be estimated as $\Delta=\left(\epsilon_{\max }-\epsilon_{\min }\right) / 2 \epsilon_{p}$, which gives

$$
\Delta=\sqrt{1+2 \frac{m_{p} c^{2}}{\epsilon_{p}}} \sqrt{\frac{\epsilon_{s}}{m_{p} c^{2}}\left(\frac{\epsilon_{s}}{m_{p} c^{2}}+2\right)} .
$$

For the currently available laser intensity $\sim 10^{20} \mathrm{~W} / \mathrm{cm}^{2}$, the obtained ion energies are less than $100 \mathrm{MeV}$, i.e., $\epsilon_{p, s} \ll$ $m_{p} c^{2}$. Thus, Eq. (1) can be approximated as $\epsilon_{\max } \approx \epsilon_{p}+\epsilon_{s}+$ $2 \sqrt{\epsilon_{p} \epsilon_{s}}$. Meanwhile, $\Delta \approx 2 \sqrt{\epsilon_{s} / \epsilon_{p}}$, which means a sufficient RPA helps reduce the energy spread, while the MSA broadens the spectrum.

\section{SIMULATION RESULTS}

As a demonstration of our theoretical model, 2D PIC simulations are performed using the EPOCH code [47]. A pure hydrogen foil with an electron density of $n_{e}=200 n_{c}$ and thickness of $22 \mathrm{~nm}$ is irradiated by a CP laser pulse with $a_{0}=10, \tau_{L}=15 T_{0}$, and $\lambda=800 \mathrm{~nm}$. Here, the target is thicker than the value given by the optimal condition of RPA (i.e., $l_{\mathrm{op}}=a_{0} n_{c} \lambda / \pi n_{e}$, about $14 \mathrm{~nm}$ ) [33] to maintain the bulk acceleration during the whole laser-plasma interaction. To better compare with the theory, the laser pulse has transversely fourth-order super-Gaussian profiles with a spot radius $r=5 \mu \mathrm{m}$ and temporally flattop envelope ( $1 T_{0}$ rise and fall times and $14 T_{0}$ plateau). This ensures the Rayleigh length is much larger than the acceleration distance in each simulation, and therefore the results can be used to estimate the upper limit of the attainable maximum energies. In other words, further increasing the laser spot size will not bring an increase of the maximum energy. The simulation box $(x, y)$ is $14.4 \mu \mathrm{m} \times 24 \mu \mathrm{m}$ containing $14400 \times 4000$ cells. Each cell contains 400 macroelectrons and 400 macroprotons.

Figure 2 shows the simulation results. Comparing Figs. 2(a) and 2(b), one can see that with a thicker target, the accelerating foil can stay opaque until the pulse is over $\left(t=18 T_{0}\right)$. Here, $t=0$ corresponds to the pulse on the target. This ensures that the LS acceleration dominates when the pulse is on, which helps maintain the energy spread to be small [black line in Fig. 2(c)]. At this stage, as shown in Fig. 2(d), the maximum (blue asterisks) and peak (blue crosses) energies 

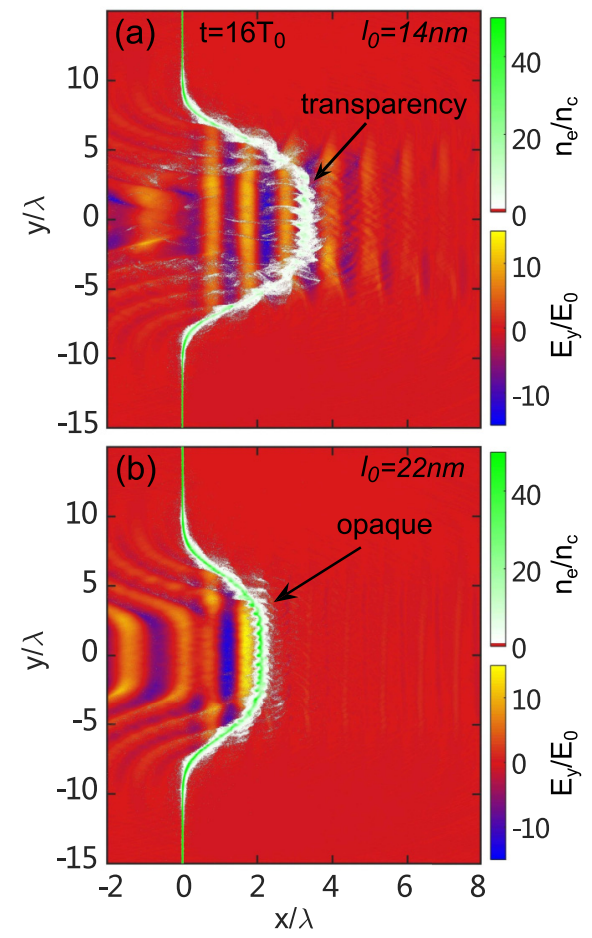
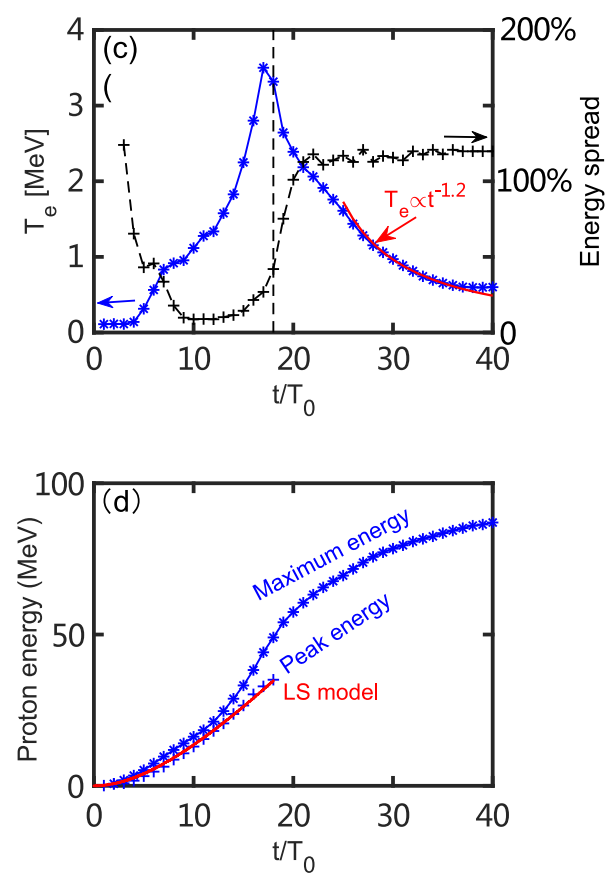
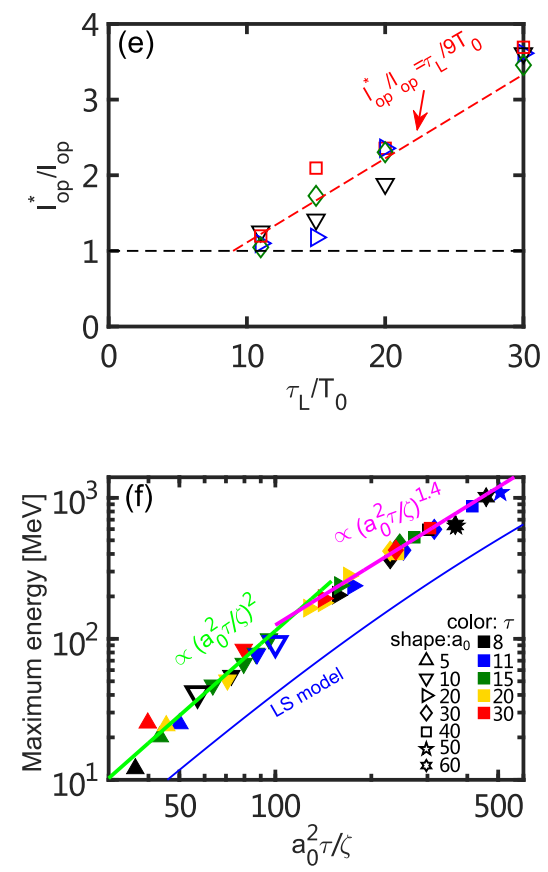

FIG. 2. (a), (b) Distributions of electron density and laser field at $t=16 T_{0}$ for the target thicknesses 14 and $22 \mathrm{~nm}$, respectively. (c) Time evolutions of electron temperature (blue) and energy spread of protons (black) for the case with a 22-nm-thick target. (d) Time evolutions of proton energies. (e) The modified optimal target thickness $l_{\mathrm{op}}^{*} / l_{\mathrm{op}}$ vs the pulse duration to maintain the bulk RPA over the whole pulse duration. (f) The maximum proton energy as a function of $a_{0}^{2} \tau / \zeta$. The open triangles represent the 3D PIC simulation results. In (e) and (f), the symbol shapes represent the laser intensity, given by the legend of (f). In (f), the pulse durations are shown by different colors.

almost coincide with each other and follow the prediction of the LS model (red line).

However, due to the transverse instabilities, the electron temperature $T_{e}$ keeps increasing up to the maximum of $T_{\max }=$ $3.5 \mathrm{MeV}$ at $t=17 T_{0}$, shown by the blue line in Fig. 2(c). This value is comparable to that given by the ponderomotive scaling 4.5 MeV [48], which indicates that significant electron heating happens even for a $\mathrm{CP}$, femtosecond laser pulse. When the laser is over, the bulk acceleration terminates and the MSA induced by hot electrons dominates, with a sheath field $E_{\text {sim }} \approx 3.7 E_{0}\left(E_{0}=m_{e} \omega c / e\right)$ [46]. This is broadly consistent with Mora's model [5] where the sheath field $E_{\mathrm{sh}}=$ $\sqrt{2 / e_{\mathrm{N}}} T_{\max } / e \lambda_{d} \approx 4.9 E_{0}$, with $e_{\mathrm{N}} \approx 2.718$ and Debye length $\lambda_{d}=\sqrt{T_{\max } / 4 \pi n_{e} e^{2}}$. Subsequently, since electron heating no longer occurs, the MSA enters into the adiabatic phase [5], where $T_{e}$ drops gradually as $T_{e} \propto t^{-1.2}$ [red line in Fig. 2(c)].

Further, to determine the coefficient $\alpha$, we carried out a series of 2D PIC simulations. The results are shown in Fig. 2(f), where the laser intensity varies from $I_{0}=10^{20}\left(a_{0}=5\right)$ to $1.5 \times 10^{22}\left(a_{0}=60\right) \mathrm{W} / \mathrm{cm}^{2}$ (seven samples represented by different shapes) and the pulse duration changes from $\tau_{L}=$ $8 T_{0}$ to $30 T_{0}$ (five samples represented by different colors). For each case, the corresponding target thickness is assigned correspondingly to the modified optimal condition $l_{\mathrm{op}}^{*}$ of RPA to maintain the bulk acceleration lasts until the end of laserplasma interaction,

$$
l_{\mathrm{op}}^{*}=l_{\mathrm{op}}(\tau / 9)
$$

which is obtained by fitting the simulation results, shown in Fig. 2(e). In this regime, the difference of the maximum energy observed in 2D and 3D simulations is small, as discussed in Refs. [29,42,49]. To further check this, we performed two 3D simulations, as shown by the open triangles in Fig. 2(f), considering that a full 3D parameter investigation is far beyond the capability of our computational resources.

By considering the results from this parameter scan, we find the coefficient $\alpha=0.84 \ln (\tau)$, which leads to the best fit of the maximum proton energies predicted by Eq. (1) with all the simulation results. A detailed comparison can be found in Supplemental Fig. S2 [46]. For example, for the case of Fig. 2(b), according to Eq. (1), $\epsilon_{\max }=73 \mathrm{MeV}$, which matches well with the simulation results $79 \mathrm{MeV}$, while the LS model predicts $32 \mathrm{MeV}$. The additional energy comes from the significant contribution of the MSA process, especially the third term (about $33 \mathrm{MeV}$ ), while the "pure" TNSA (the second term) only contributes about $8 \mathrm{MeV}$.

Substituting $\alpha$ to Eq. (2), we have $\Delta \approx 2 \sqrt{\epsilon_{s} / \epsilon_{p}} \approx$ $1.97 \sqrt{\ln (\tau) / a_{0}}$. This indicates that, with larger $a_{0}$ or smaller $\tau$, the energy spread is lower. For the currently achievable femtosecond lasers, typically $a_{0} \approx 10$ and $\tau \approx 15$, one has $\Delta \approx 103 \%$. This explains why so far the experimentally measured ion energy spectrum is always broad even for the cases where RPA is dominant [13,25,28,29].

More practical scaling laws, just related to the initial laser and plasma parameters, can be given by fitting the maximum ion energy $\epsilon_{\max }$ and parameters $a_{0}^{2} \tau / \zeta$ from the 2D simulation results in Fig. 2(f). In different energy ranges, corresponding 
to the nonrelativistic and relativistic regimes, as shown by the solid green and magenta lines, the scaling laws are respectively given as

$$
\begin{array}{rlrl}
\epsilon_{n r} & \approx 0.0114 \frac{Z}{A}\left(a_{0}^{2} \tau / \zeta\right)^{2.0}[\mathrm{MeV} / \mu], & a_{0}^{2} \tau / \zeta<150, \\
\epsilon_{r} \approx 0.1984 \frac{Z}{A}\left(a_{0}^{2} \tau / \zeta\right)^{1.4}[\mathrm{MeV} / \mu], & a_{0}^{2} \tau / \zeta>150 .
\end{array}
$$

For $a_{0}^{2} \tau / \zeta<150$ (the normalized ion velocity $\beta_{p}<0.5$ ), corresponding to the nonrelativistic regime, the ion maximum energy obeys the scaling Eq. (4a). When $a_{0}^{2} \tau / \zeta>150, \beta_{p}>$ 0.5 , the ion relativistic effect becomes important since $\xi$ cannot be ignored in the denominator of $\epsilon_{p}$ in the LS model. This results in a slower scaling of Eq. (4b). In the ultrarelativistic case, where $a_{0}^{2} \tau / \zeta \gg m_{p} / m_{e}$, the scaling further degrades to $\epsilon_{p} \propto a_{0}^{2} \tau / \zeta$

If we substitute the modified optimal condition $l_{\mathrm{op}}^{*}$ into Eqs. (4a) and (4b), we yield the scaling laws just related to laser intensity,

$$
\begin{aligned}
\epsilon_{n r} & \approx 0.92 \frac{Z}{A} a_{0}^{2.0}[\mathrm{MeV} / \mu] \propto I_{0}, \quad a_{0}^{2} \tau / \zeta<150, \\
\epsilon_{r} & \approx 4.30 \frac{Z}{A} a_{0}^{1.4}[\mathrm{MeV} / \mu] \propto I_{0}^{0.7}, \quad a_{0}^{2} \tau / \zeta>150 .
\end{aligned}
$$

These scaling laws represent the upper limit of the maximum energy that ions can obtain from intense laser-irradiated nanometer-scale foil targets. For example, for $a_{0}=10$ and $a_{0}=30$, Eqs. (5a) and (5b) give the maximum energies of 92 and $503 \mathrm{MeV}$, respectively, which are in good agreement with the simulation results, as shown in Fig. 2(f) with the solid inverted triangles and the solid diamonds.

\section{COMPARISON WITH PUBLISHED EXPERIMENTAL RESULTS}

To validate the scaling laws of Eqs. (4) and (5), we take the published experimental data of ion acceleration from $\mathrm{nm}$-scale ultrathin foils from various facilities for comparison [13,2530,50-53]. To the best of our knowledge, we have included the most representative results. Considering that in these experiments the target bulk mostly consists of carbon or metallic ions, we first compare the predictions of Eq. (4a) with the energies observed in RPA-dominated experiments (circles in Fig. 3). The red circles represent the maximum energies of $\mathrm{C}^{6+}$ obtained from Refs. $[25,28,29]$ and the blue one stands for that of $\mathrm{Cu}^{27+}$ from Ref. [13]. Meanwhile the dashed red and blue lines correspond to predictions from Eq. (4a) for $\mathrm{C}^{6+}$ and $\mathrm{Cu}^{27+}$ ions, respectively. One can clearly see that the scaling (4a) is broadly consistent with all the experimental results, considering that a linear-scale $y$ axis is used here. Note that the number of the comparison samples is limited by the experimental results reported at this regime.

In Fig. 4, we further compare the experimental results of protons with the scaling laws of Eq. (5). Considering that in present ultrathin foil experiments, the maximum ion energies obtained from CP lasers are always comparable or higher than those from LP lasers [28,29], Eq. (5) actually predicts the upper limit of the achievable maximum energy that ions can be obtained from given laser parameters, regardless of the laser polarization. Therefore, in Fig. 4, the experimental

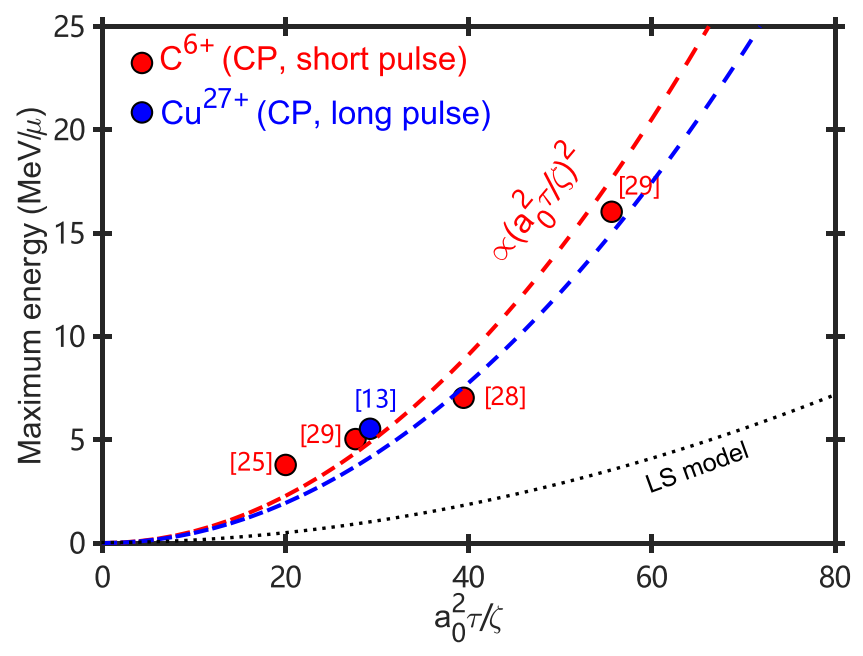

FIG. 3. Comparison of the reported RPA-dominated experimental results (shown by the reference number) and the scaling law [Eq. (4a)]. The red circles and dashed line represent the experimental data and scaling law for $\mathrm{C}^{6+}$, respectively, while the blue ones correspond to $\mathrm{Cu}^{27+}$. The black dotted line depicts the predictions of the LS model.

results with LP lasers are also included. One can see that on the one hand, most of the experimental results are lower than the red line; on the other hand, the upper limits of proton energies obtained from experiments at different laser intensities act in good accordance with the predictions of Eq. (5). By comparing with the experimental results reported in Refs. $[25,30,50]$ marked by larger symbols in Fig. 4, one can see a clear trend that follows our prediction. This indicates that one can use Eq. (5) to estimate the maximum attainable ion energy for the future experiments. Note that for the experimental results at higher laser intensities $\left(I_{0} \lambda^{2}>\right.$ $\left.6 \times 10^{20} \mathrm{~W} \mu \mathrm{m}^{2} / \mathrm{cm}^{2}\right)[26,52]$ shown in Fig. 4, the obtained proton energies are much lower than the predictions. This is

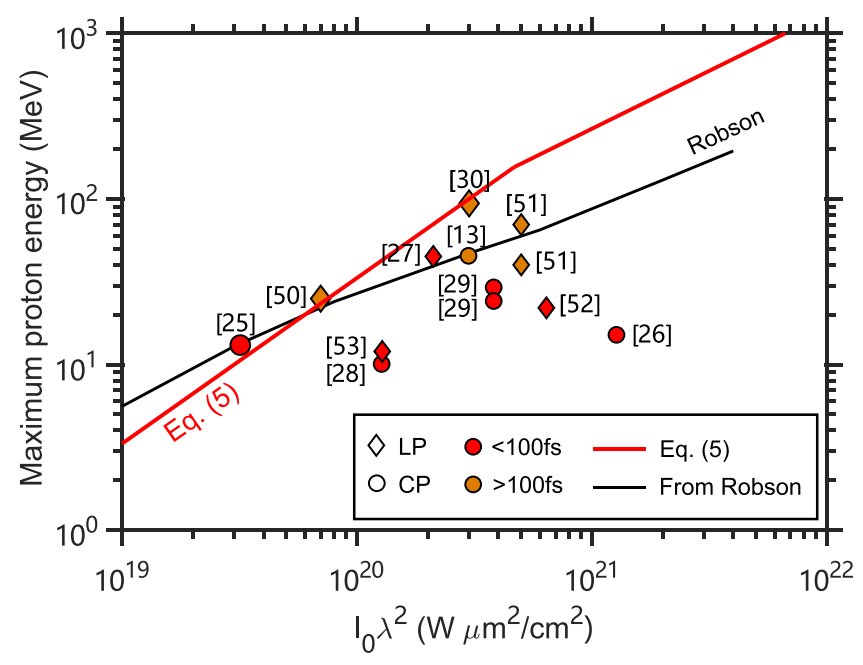

FIG. 4. Comparison of the reported experimental results (maximum proton energy) from laser-irradiating nanofoils (shown by the reference number) and the scaling law [Eq. (5), red line]. The black line is the scaling of TNSA from Ref. [8]. 
because the operating laser and plasma parameters were far from optimal since very small focal spot sizes were used to achieve high laser intensities. The bulk acceleration terminates very early due to the serious electron heating, leading to much smaller energies.

\section{CONCLUSION}

In summary, we have derived scaling laws for the attainable ion-beam maximum energy from laser-irradiated nanometerscale ultrathin foil targets. The scaling laws are demonstrated by $2 \mathrm{D}$ and $3 \mathrm{D}$ simulations and, more importantly, coincide well with relevant experimental data from different facilities over a large range of laser and target parameters. This provides important information for future experimental design and laser facility construction. In the context of a prospective application of laser-driven protons for future cancer therapy, the production of $200-\mathrm{MeV}$ protons would be an important milestone as this is the energy which allows reaching deep seated tumors in the body. Using the scaling of Eq. (4), we can deduce that for an ultrathin foil target with an areal density of about $2 \times 10^{22} \mathrm{~m}^{-2}$, to obtain a proton beam with a maximum energy of $200 \mathrm{MeV}$, an intense laser pulse with an intensity of about $2 \times 10^{21} \mathrm{~W} / \mathrm{cm}^{2}$ and a pulse duration of around $40 \mathrm{fs}$ is required, which means the laser energy is at least $70 \mathrm{~J}$ considering a laser pulse with a spot radius of $4 \mu \mathrm{m}$ to avoid the finite spot size effects. Such laser parameters are at the limit of the capacity for present femtosecond laser systems (particularly if double plasma mirrors are employed, which reduce the energy delivered on target), but would be soon attained in upcoming multipetawatt laser facilities $[54,55]$.

\section{ACKNOWLEDGMENTS}

This work was supported by Science Challenge Project, No. TZ2018005; National Natural Science Foundation of China, Grants No. 11825502, No. 11921106, and No. 12075014; the Strategic Priority Research Program of Chinese Academy of Sciences Grant No. XDA25050900; the National Key R\&D Program of China, Grant No. 2016YFA0401100; and the EPSRC, Grant No. EP/K022414/1. B.Q. acknowledges support from National Natural Science Funds for Distinguished Young Scholar, Grant No. 11825502. X.F.S. gratefully acknowledges support by the Alexander von Humboldt Foundation. X.F.S. appreciates Dr. X. B. Li, J. Wang, Y. Xie, Dr. J. H. Bin, Dr. C. Scullion, and Dr. D. Doria for useful discussions. The computational resources are supported by the Special Program for Applied Research on Super Computation of the NSFC-Guangdong Joint Fund (the second phase) and GCS Jülich (project QED20).
[1] A. Macchi, M. Borghesi, and M. Passoni, Ion acceleration by superintense laser-plasma interaction, Rev. Mod. Phys. 85, 751 (2013).

[2] H. Daido, M. Nishiuchi, and A. S. Pirozhkov, Review of laserdriven ion sources and their applications, Rep. Prog. Phys. 75, 056401 (2012).

[3] R. A. Snavely, M. H. Key, S. P. Hatchett, T. E. Cowan, M. Roth, T. W. Phillips, M. A. Stoyer, E. A. Henry, T. C. Sangster, M. S. Singh, S. C. Wilks, A. MacKinnon, A. Offenberger, D. M. Pennington, K. Yasuike, A. B. Langdon, B. F. Lasinski, J. Johnson, M. D. Perry, and E. M. Campbell, Intense High-Energy Proton Beams from Petawatt-Laser Irradiation of Solids, Phys. Rev. Lett. 85, 2945 (2000).

[4] P. Mora, Plasma Expansion into a Vacuum, Phys. Rev. Lett. 90, 185002 (2003).

[5] P. Mora, Thin-foil expansion into a vacuum, Phys. Rev. E 72, 056401 (2005).

[6] J. Fuchs, P. Antici, E. d'Humières, E. Lefebvre, M. Borghesi, E. Brambrink, C. A. Cecchetti, M. Kaluza, V. Malka, M. Manclossi et al., Laser-driven proton scaling laws and new paths towards energy increase, Nat. Phys. 2, 48 (2006).

[7] J. Schreiber, F. Bell, F. Grüner, U. Schramm, M. Geissler, M. Schnürer, S. Ter-Avetisyan, B. M. Hegelich, J. Cobble, E. Brambrink et al., Analytical Model for Ion Acceleration by High-Intensity Laser Pulses, Phys. Rev. Lett. 97, 045005 (2006).

[8] L. Robson, P. T. Simpson, R. J. Clarke, K. W. D. Ledingham, F. Lindau, O. Lundh, T. McCanny, P. Mora, D. Neely, C.G. Wahlström, M. Zepf et al., Scaling of proton acceleration driven by petawatt-laser-plasma interactions, Nat. Phys. 3, 58 (2007).
[9] M. Borghesi, A. Bigongiari, S. Kar, A. Macchi, L. Romagnani, P. Audebert, J. Fuchs, T. Toncian, O. Willi, S. V. Bulanov et al., Laser-driven proton acceleration: source optimization and radiographic applications, Plasma Phys. Control. Fusion 50, 124040 (2008).

[10] K. Zeil, S. D. Kraft, S. Bock, M. Bussmann, T. E. Cowan, T. Kluge, J. Metzkes, T. Richter, R. Sauerbrey, and U. Schramm, The scaling of proton energies in ultrashort pulse laser plasma acceleration, New J. Phys. 12, 045015 (2010).

[11] M. Nakatsutsumi, Y. Sentoku, A. Korzhimanov, S. N. Chen, S. Buffechoux, A. Kon, B. Atherton, P. Audebert, M. Geissel, L. Hurd et al., Self-generated surface magnetic fields inhibit laser-driven sheath acceleration of high-energy protons, Nat. Commun. 9, 280 (2018).

[12] T. Esirkepov, M. Yamagiwa, and T. Tajima, Laser IonAcceleration Scaling Laws Seen in Multiparametric Particle-inCell Simulations, Phys. Rev. Lett. 96, 105001 (2006).

[13] S. Kar, K. F. Kakolee, B. Qiao, A. Macchi, M. Cerchez, D. Doria, M. Geissler, P. McKenna, D. Neely, J. Osterholz et $a l$., Ion Acceleration in Multispecies Targets Driven by Intense Laser Radiation Pressure, Phys. Rev. Lett. 109, 185006 (2012).

[14] M. Borghesi, Laser-driven ion acceleration: State of the art and emerging mechanisms, Nucl. Instrum. Methods Phys. Res., Sect. A 740, 6 (2014).

[15] B. Qiao, X. F. Shen, H. He, Y. Xie, H. Zhang, C. T. Zhou, S. P. Zhu, and X. T. He, Revisit on ion acceleration mechanisms in solid targets driven by intense laser pulses, Plasma Phys. Controlled Fusion 61, 014039 (2019).

[16] X. F. Shen, B. Qiao, H. Zhang, Y. Xie, S. Kar, M. Borghesi, M. Zepf, C. T. Zhou, S. P. Zhu, and X. T. He, Electrostatic 
capacitance-type acceleration of ions with an intense few-cycle laser pulse, Appl. Phys. Lett. 114, 144102 (2019).

[17] W. J. Ma, I. J. Kim, J. Q. Yu, I. W. Choi, P. K. Singh, H. W. Lee, J. H. Sung, S. K. Lee, C. Lin, Q. Liao et al., Laser Acceleration of Highly Energetic Carbon Ions using a Double-Layer Target Composed of Slightly Underdense Plasma and Ultrathin Foil, Phys. Rev. Lett. 122, 014803 (2019).

[18] S. V. Bulanov, T. Z. Esirkepov, V. S. Khoroshkov, A. V. Kuznetsov, and F. Pegoraro, Oncological hadrontherapy with laser ion accelerators, Phys. Lett. A 299, 240 (2002).

[19] M. Borghesi, A. Schiavi, D. H. Campbell, M. G. Haines, O. Willi, A. J. MacKinnon, L. A. Gizzi, M. Galimberti, R. J. Clarke, and H. Ruhl, Proton imaging: A diagnostic for inertial confinement fusion/fast ignitor studies, Plasma Phys. Controlled Fusion 43, A267 (2001).

[20] M. Roth, T. E. Cowan, M. H. Key, S. P. Hatchett, C. Brown, W. Fountain, J. Johnson, D. M. Pennington, R. A. Snavely, S. C. Wilks et al., Fast Ignition by Intense Laser-Accelerated Proton Beams, Phys. Rev. Lett. 86, 436 (2001).

[21] S. C. Wilks, A. B. Langdon, T. E. Cowan, M. Roth, M. Singh, S. Hatchett, M. H. Key, D. Pennington, A. MacKinnon, and R. A. Snavely, Energetic proton generation in ultra-intense laser-solid interactions, Phys. Plasmas 8, 542 (2001).

[22] F. Wagner, O. Deppert, C. Brabetz, P. Fiala, A. Kleinschmidt, P. Poth, V. A. Schanz, A. Tebartz, B. Zielbauer, M. Roth et al., Maximum Proton Energy above $85 \mathrm{MeV}$ from the Relativistic Interaction of Laser Pulses with Micrometer Thick $\mathrm{CH}_{2}$ Targets, Phys. Rev. Lett. 116, 205002 (2016).

[23] A. J. Mackinnon, Y. Sentoku, P. K. Patel, D. W. Price, S. Hatchett, M. H. Key, C. Andersen, R. Snavely, and R. R. Freeman, Enhancement of Proton Acceleration by Hot-Electron Recirculation in Thin Foils Irradiated by Ultraintense Laser Pulses, Phys. Rev. Lett. 88, 215006 (2002).

[24] M. Kaluza, J. Schreiber, M. I. K. Santala, G. D. Tsakiris, K. Eidmann, J. Meyer-ter-Vehn, and K. J. Witte, Influence of the Laser Prepulse on Proton Acceleration in Thin-Foil Experiments, Phys. Rev. Lett. 93, 045003 (2004).

[25] A. Henig, S. Steinke, M. Schnürer, T. Sokollik, R. Hörlein, D. Kiefer, D. Jung, J. Schreiber, B. M. Hegelich, X. Q. Yan et al., Radiation-Pressure Acceleration of Ion Beams Driven by Circularly Polarized Laser Pulses, Phys. Rev. Lett. 103, 245003 (2009).

[26] F. Dollar, C. Zulick, A. G. R. Thomas, V. Chvykov, J. Davis, G. Kalinchenko, T. Matsuoka, C. McGuffey, G. M. Petrov, L. Willingale, V. Yanovsky et al., Finite Spot Effects on Radiation Pressure Acceleration from Intense High-Contrast Laser Interactions with Thin Targets, Phys. Rev. Lett. 108, 175005 (2012).

[27] I. J. Kim, K. H. Pae, C. M. Kim, H. T. Kim, J. H. Sung, S. K. Lee, T. J. Yu, I. W. Choi, C.-L. Lee, K. H. Nam, P. V. Nickles et al., Transition of Proton Energy Scaling using an Ultrathin Target Irradiated by Linearly Polarized Femtosecond Laser Pulses, Phys. Rev. Lett. 111, 165003 (2013).

[28] J. H. Bin, W. J. Ma, H. Y. Wang, M. J. V. Streeter, C. Kreuzer, D. Kiefer, M. Yeung, S. Cousens, P. S. Foster, B. Dromey et al., Ion Acceleration using Relativistic Pulse Shaping in Near-Critical-Density Plasmas, Phys. Rev. Lett. 115, 064801 (2015).

[29] C. Scullion, D. Doria, L. Romagnani, A. Sgattoni, K. Naughton, D. R. Symes, P. McKenna, A. Macchi, M. Zepf, S. Kar et al.,
Polarization Dependence of Bulk Ion Acceleration from Ultrathin Foils Irradiated by High-Intensity Ultrashort Laser Pulses, Phys. Rev. Lett. 119, 054801 (2017).

[30] A. Higginson, R. J. Gray, M. King, R. J. Dance, S. D. R. Williamson, N. M. H. Butler, R. Wilson, R. Capdessus, C. Armstrong, J. S. Green et al., Near-100 MeV protons via a laserdriven transparency-enhanced hybrid acceleration scheme, Nat. Commun. 9, 724 (2018).

[31] T. Esirkepov, M. Borghesi, S. V. Bulanov, G. Mourou, and T. Tajima, Highly Efficient Relativistic-Ion Generation in the Laser-Piston Regime, Phys. Rev. Lett. 92, 175003 (2004).

[32] A. P. L. Robinson, M. Zepf, S. Kar, R. G. Evans, and C. Bellei, Radiation pressure acceleration of thin foils with circularly polarized laser pulses, New J. Phys. 10, 013021 (2008).

[33] A. Macchi, S. Veghini, and F. Pegoraro, "Light Sail" Acceleration Reexamined, Phys. Rev. Lett. 103, 085003 (2009).

[34] B. Qiao, M. Zepf, M. Borghesi, and M. Geissler, Stable GeV Ion-Beam Acceleration from Thin Foils by Circularly Polarized Laser Pulses, Phys. Rev. Lett. 102, 145002 (2009).

[35] O. Klimo, J. Psikal, J. Limpouch, and V. T. Tikhonchuk, Monoenergetic ion beams from ultrathin foils irradiated by ultrahigh-contrast circularly polarized laser pulses, Phys. Rev. Accel. Beams 11, 031301 (2008).

[36] A. Macchi, S. Veghini, T. V. Liseykina, and F. Pegoraro, Radiation pressure acceleration of ultrathin foils, New J. Phys. 12, 045013 (2010).

[37] M. Grech, S. Skupin, A. Diaw, T.Schlegel, and V. T. Tikhonchuk, Energy dispersion in radiation pressure accelerated ion beams, New J. Phys. 13, 123003 (2011).

[38] B. Qiao, S. Kar, M. Geissler, P. Gibbon, M. Zepf, and M. Borghesi, Dominance of Radiation Pressure in Ion Acceleration with Linearly Polarized Pulses at Intensities of $1021 \mathrm{~W} / \mathrm{cm}^{2}$, Phys. Rev. Lett. 108, 115002 (2012).

[39] F. Pegoraro and S. V. Bulanov, Photon Bubbles and Ion Acceleration in a Plasma Dominated by the Radiation Pressure of an Electromagnetic Pulse, Phys. Rev. Lett. 99, 065002 (2007).

[40] E. Siminos, M. Grech, S. Skupin, T. Schlegel, and V. T. Tikhonchuk, Effect of electron heating on self-induced transparency in relativistic-intensity laser-plasma interactions, Phys. Rev. E 86, 056404 (2012).

[41] A. Sgattoni, S. Sinigardi, L. Fedeli, F. Pegoraro, and A. Macchi, Laser-driven Rayleigh-Taylor instability: Plasmonic effects and three-dimensional structures, Phys. Rev. E 91, 013106 (2015).

[42] X. F. Shen, B. Qiao, H. Zhang, S. Kar, C. T. Zhou, H. X. Chang, M. Borghesi, and X. T. He, Achieving Stable Radiation Pressure Acceleration of Heavy Ions via Successive Electron Replenishment from Ionization of a High-Z Material Coating, Phys. Rev. Lett. 118, 204802 (2017).

[43] X. F. Shen, B. Qiao, H. X. Chang, W. L. Zhang, H. Zhang, C. T. Zhou, and X. T. He, Maintaining stable radiation pressure acceleration of ion beams via cascaded electron replenishment, New J. Phys. 19, 033034 (2017).

[44] X. Q. Yan, H. C. Wu, Z. M. Sheng, J. E. Chen, and J. Meyer-terVehn, Self-Organizing GeV, Nanocoulomb, Collimated Proton Beam from Laser Foil Interaction at $7 \times 10^{21} \mathrm{~W} / \mathrm{cm}^{2}$, Phys. Rev. Lett. 103, 135001 (2009).

[45] A. Pukhov, Three-Dimensional Simulations of Ion Acceleration from a Foil Irradiated by a Short-Pulse Laser, Phys. Rev. Lett. 86, 3562 (2001). 
[46] See Supplemental Material at http://link.aps.org/supplemental/ 10.1103/PhysRevE.104.025210 for details of the distributions of the longitudinal electric field and a comparison of simulation results with theoretical predictions.

[47] T. D. Arber, K. Bennett, C. S. Brady, A. Lawrence-Douglas, M. G. Ramsay, N. J. Sircombe, P. Gillies, R. G. Evans4, H. Schmitz, A. R. Bell et al., Contemporary particle-in-cell approach to laser-plasma modelling, Plasma Phys. Controlled Fusion 57, 113001 (2015).

[48] S. C. Wilks, W. L. Kruer, M. Tabak, and A. B. Langdon, Absorption of Ultra-Intense Laser Pulses, Phys. Rev. Lett. 69, 1383 (1992).

[49] A. Sgattoni, S. Sinigardi, and A. Macchi, High energy gain in three-dimensional simulations of light sail acceleration, Appl. Phys. Lett. 105, 084105 (2014).

[50] A. Henig, D. Kiefer, K. Markey, D. C. Gautier, K. A. Flippo, S. Letzring, R. P. Johnson, T. Shimada, L. Yin, B. J. Albright et al., Enhanced Laser-Driven Ion Acceleration in the Relativistic Transparency Regime, Phys. Rev. Lett. 103, 045002 (2009).
[51] D. Jung, B. J. Albright, L. Yin, D. C. Gautier, R. Shah, S. Palaniyappan, S. Letzring, B. Dromey, H.-C. Wu, T. Shimada et al., Beam profiles of proton and carbon ions in the relativistic transparency regime, New J. Phys. 15, 123035 (2013).

[52] P. L. Poole, L. Obst, G. E. Cochran, J. Metzkes, H.-P. Schlenvoigt, I. Prencipe, T. Kluge, T. Cowan, U. Schramm, D. W. Schumacher et al., Laser-driven ion acceleration via target normal sheath acceleration in the relativistic transparency regime, New J. Phys. 20, 013019 (2018).

[53] J. H. Bin, M. Yeung, Z. Gong, H. Y. Wang, C. Kreuzer, M. L. Zhou, M. J. V. Streeter, P. S. Foster, S. Cousens, B. Dromey et al., Enhanced Laser-Driven Ion Acceleration by Superponderomotive Electrons Generated from Near-CriticalDensity Plasma, Phys. Rev. Lett. 120, 074801 (2018).

[54] G. Mourou and T. Tajima, The extreme light infrastructure: optics' next horizon, Opt. Photonics News 22, 47 (2011).

[55] C. Danson, D. Hillier, N. Hopps, and D. Neely, Petawatt class lasers worldwide, High Power Laser Sci. Eng. 3, e3 (2015). 